\title{
Centralised versus Decentralised Traffic Optimisation of Urban Road Networks: A Simulation Study
}

\author{
Mauro Vallati \\ School of Computing \& Engineering, \\ University of Huddersfield, \\ Huddersfield, United Kingdom \\ m.vallati@hud.ac.uk
}

\begin{abstract}
One of the pivotal challenges presented to urban road traffic controllers is the effective utilisation of transport infrastructure, as a result of growing urbanisation, the finite network capacity, and of the increasing number of road vehicles. In this context, the arrival of connected autonomous vehicles (CAVs) represents a unique opportunity for a fundamental change in urban traffic optimisation, and urban traffic control should take an active role in integrating CAVs into the mobility ecosystem in order to maximise benefits.

Traditional approaches, commonly exploited by SATNAVs, are based on a decentralised logic, where each vehicle decides the route to follow in isolation, possibly by considering the current network conditions. The arrival of connected vehicles would allow the exploitation of centralised traffic optimisation, where a central urban traffic controller can suggest routes to vehicles by taking into account the current network conditions, and predicted future evolution. This paper introduces a centralised approach for traffic optimisation of urban road networks, and presents an extensive evaluation of the capabilities of centralised and decentralised approaches. Evaluation is based on a validated and calibrated SUMO simulation model of the town centre of Milton Keynes, United Kingdom.
\end{abstract}

Keywords-Urban Traffic Control; Traffic Optimisation; Centralised vs Decentralised

\section{INTRODUCTION}

Over half of the world's population now lives in cities and global urbanisation continues at a steady pace. In this context, the arrival of Connected Autonomous Vehicles $(\mathrm{CAVs})$ presents a unique opportunity for a fundamental change in urban mobility and urban traffic control. In particular, CAVs can communicate with other vehicles and with the infrastructure, via dedicated protocols, to take better informed decisions. While the general importance and improved capabilities that can be obtained via different types of communication have been well argued [1], and a number of protocols and technologies to implement such communications has been presented [2], here we focus on how urban traffic control can leverage on connected vehicles to perform traffic optimisation. Nowadays, it is often the case that during rush hours most main roads of a urban region are congested, at least in one direction, while many other roads are underused. Such under-use of the network is caused by the fact that traffic often navigates via the same route between given way-points, as the result of similar behaviour and habits of vehicle drivers. Considering the available network, it is often the case that the exploitation of alternative routes can lead to a better distribution of vehicles and a better use of the capacity of the network.

Existing approaches to traffic optimisation are mostly decentralised: vehicles are equipped with satellite navigators that can suggest routes on the basis of real-time traffic conditions. The arrival of CAVs can support the use of centralised traffic optimisation approaches, where a urban traffic controller can suggest the best route to follow for a given vehicle, taking into account the overall network conditions, the paths of vehicles navigating in the area, and the predicted evolution of the traffic. Further, centralised approaches can consider a wide range of metrics for optimising the conditions of the network. As for different areas of urban traffic control, such as signal control [3], it is of paramount importance to understand the weaknesses and strengths of techniques based on either centralised or decentralised logic, to foster the progress in the field.

The aim of this paper is to investigate, under realistic traffic conditions, the effectiveness and performance of approaches based on centralised and decentralised logic for performing traffic optimisation in the busy modern urban areas. As a decentralised approach, the traditional technique based on real-time travel times is considered. We then introduce a framework that exploits an innovative centralised approach based on the notion of cumulative occupancy. Connected vehicles in the controlled area communicate their destination and current route to a controller, that, given its understanding of the current and predicted status of the network, can decide whether to propose an alternative route to a vehicle. The centralised and decentralised strategies are compared in simulation, using real-world traffic data of the Milton Keynes centre area.

\section{RELATED WORKS}

SatNav systems, particularly under the form of smartphone navigator apps, are commonly used in the industry and by everyday road users. Well-known examples include 
Waze ${ }^{1}$ and Navfree ${ }^{2}$ They do take a decentralised (distributed) approach, where each vehicle is trying to optimise its own path considering the current network condition, that is usually assessed in terms of travel times. There is no cooperation among vehicles.

Ramamohanarao et al. [4] discussed how CAVs capabilities can be integrated with smart systems to enhance traffic optimisation. This includes vehicle-to-vehicle cooperation, as well as cooperation between vehicles and infrastructure, for instance by calibrating traffic light phases according to incoming vehicles.

Jimoh et al. [5] studied the use of AI-based automated planning for dealing with situations where some roads within an urban road network become blocked due to some unanticipated event (e.g. floods). Jimoh et al. demonstrated the feasibility of the current AI planning technology to address this issue on a small scale; the proposed approach is able to produce plans for re-routing tens of vehicles using a simple yet effective micro-simulation model. The work of Chrpa et al. [6] exploits AI-based automated planning techniques for enforcing air-quality constraints in urban traffic. In a more recent work, Chrpa et al. [7], showed that AI-based planning techniques can be used for reducing congestion of a controlled urban region by re-routing vehicles, but their work is at an exploratory stage and can cope only with a very limited number of vehicles.

Jeong et al. [8] considered collaborative path optimisation and propose a navigation system named SAINT. In this system, a centralised navigation server monitors all navigation requests and traffic conditions reported by drivers, and suggest paths, considering a trade-off between length and congestion, using a modified Dijkstra algorithm. The authors recently introduced SAINT+ [9], that is focused on path optimisation for emergency vehicles.

Finally, Rezaei et al. [10] introduced ReFOCUS, that aims at optimally re-routing vehicles in congested areas, or approaching congested areas, by leveraging on fogcloud computing performed by vehicles. The focus of the work is on minimising the information exchanged and the complexity of the algorithms.

\section{THE CONSIDERED APPROACH}

This section is devoted to introduce the centralised architecture and approach, and to describe the considered decentralised technique that will be used for performing the comparison.

\section{A. The Centralised Controller}

Figure 11 provides an overview of a general centralised architecture that can be exploited to deal with the problem of traffic optimisation in urban regions. The centralised urban traffic controller can leverage on a significant amount of

${ }^{1}$ https://www.waze.com/

2 http://navfree.android.informer.com/
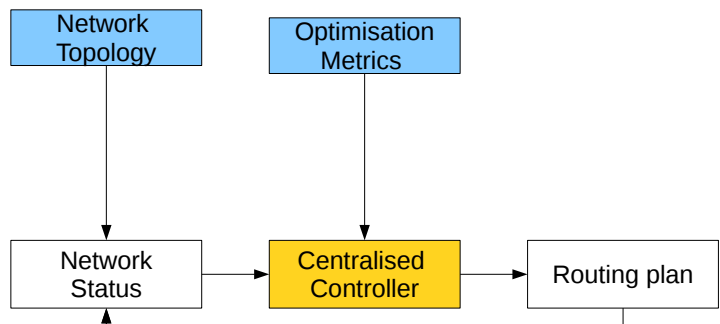

Real-time

Network data

Figure 1: An overview of the introduced centralised architecture. Blue is used to indicate data and knowledge. Yellow depicts the controller. White is used to highlight input and outputs of the controller, under the form of aggregated data.

data. The controlled network status is defined by aggregating data about:

- Network topology. This includes a description of the topology of the network in terms of links, junctions, etc.

- Real-time data. Available sensors distributed in the controlled region can provide additional information about the status, for instance in terms of air quality, congestion, accidents, etc. In fact, it is worth noting that there may be vehicles that are not connected, and therefore their presence can only be identified via traditional sensors (pressure, cameras, etc.).

- Navigating connected vehicles. Data acquired from vehicles that are navigating or approaching the controlled region include: current location, final destination, and the currently assigned path.

The defined status is then provided as input to the centralised controller that, by taking into account some predefined optimisation metrics, is in the best position for assessing the network conditions and deciding if there is the need for re-routing vehicles in order to maintain a required level of service, or to counteract to some unexpected conditions. If new routing plans are generated, they are communicated to the affected vehicles using an appropriate protocol.

Having described the overall architecture that can be implemented in order to perform centralised traffic control in the presence of connected vehicles, we are now in a position to introduce the structure of the controller itself. This is of course the pivotal module of the architecture presented in Figure 1

Algorithm 1 provides a high level description of the designed centralised controller. Lines 1-2 focus on acquiring the relevant information in terms of status of the network 


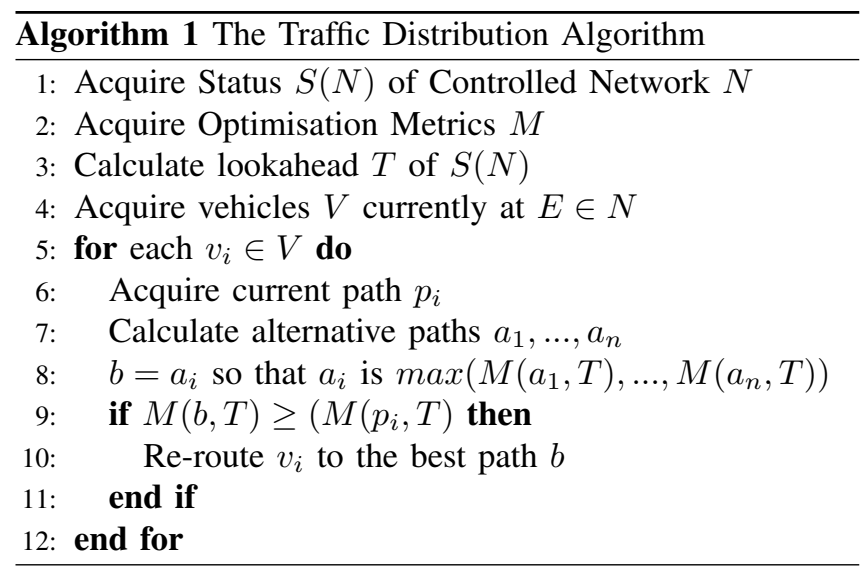

$S(N)$ and the considered optimisation metric. In Line 3, the expected future evolution of the network is calculated according to the available data. Then, in Line 4, vehicles that are currently at entry links $E$ are identified. For the sake of this analysis, and in order to minimise the burden on vehicles, we assume that vehicles can be rerouted only when they are on the boundaries of the controlled region. In other words, the controller can only consider the option of re-routing vehicles that are on links directly connected to an entry point.

Lines 5-12 contain the main loop, where for each identified vehicle that is entering the network $v_{i}$ alternative routes for the specified destination are calculated and compared. Routes are compared by taking into account the specified optimisation metric $M$ and the expected future conditions of the network. The best alternative option is assigned to the vehicle.

In this work we consider congestion as the main optimisation metric to be taken into account by the centralised controller; the controller has to distribute vehicles in the urban network in order to minimise the congestion of links. To support the ability of the controller in evaluating the future evolution of the network, and estimate the congestion of a path, we introduce the notion of cumulative occupancy.

Given a lookahead $T$ of a controlled network, the cumulative occupancy of a path $P$, considered as a sequence of links, is calculated as:

$$
c(P)=\sum_{n \in P} \text { vehicles }(n, T)
$$

Where vehicles $(n, T)$ represents the expected number of vehicles to navigate through link $n$ within the considered time horizon $T$. Remarkably, the notion of cumulative occupancy allows to reduce the computational complexity of predicting the future evolution of the network conditions. The route followed by a vehicle is considered occupied by the vehicle for the whole time. Junctions are not considered during the evaluation of cumulative occupancy, as the measure is focused on links. In other words, when a vehicle is assigned a route, all the links of the route are "booked" against the vehicle. This gives the centralised controller a quite pessimistic overview of the future status of the network, and can help in proactively searching for alternative options. Intuitively, this heuristic works well in busy conditions, where the number of vehicles that are entering the network is similar to vehicles exiting, and the network is overall busy.

\section{B. The Decentralised Approach}

The decentralised approach considered in this work is the one mostly used in smartphone navigator apps, based on the notion of estimated travel time. Given the decentralised nature of the system, the travel time estimation is made on the basis of the current condition of the network, and by considering the network topology. In particular, the travel time estimation for a path $P$, considered as a sequence of links, is calculated as:

$$
\operatorname{travel} T(P, t)=\sum_{n \in P} \operatorname{travel} T(n, t)
$$

Where travelT $(n, t)$ represents the most recent travel time measurement for link $n$, available at time $t$.

The vehicle for which the evaluation is performed is unaware about the routes followed by the other vehicles, but is only able to indirectly consider the presence of other vehicles by considering their (current) impact on the expected travel time of urban links.

Similarly to the centralised approach, we assume that vehicles can consider re-routing options only if they are at one of the entry links $E$.

\section{EMPIRICAL ANALYSIS}

The aim of this empirical evaluation is to highlight strengths and weaknesses of using centralised and decentralised traffic optimisation approaches for preventing and mitigating congestion issues in urban areas. In particular, here we focus on connected vehicles that can modify their route according to provided information.

\section{A. Considered Scenario: The Milton Keynes Urban Area}

For this analysis we considered a SUMO [11] microsimulation model of Milton Keynes centre, which includes Avebury, Midsummer, and Silbury Boulevard crossing Elder Gate (railway station, on the south west of the map) and Saxon Gate (shopping centre, on the north east of the map). The network is shown in Figure 2. Milton Keynes is a town of the United Kingdom, located about 80 kilometres northwest of London. Milton Keynes has a population of circa 230,000 . The model covers an area of approximately 2.9 square kilometres, and includes more than 25 junctions and more than 50 links. Notably, the grid-like structure of Milton Keynes centre is structurally very similar to a typical city of the United States.

As a basis for comparing centralised and decentralised urban traffic control approaches, we chose to use historical 


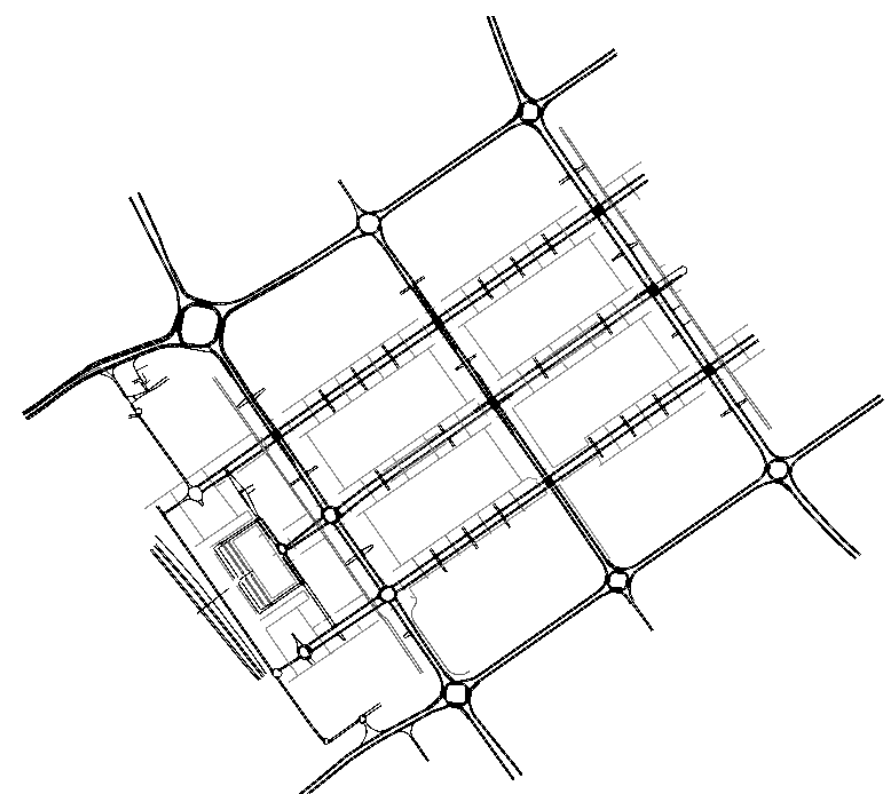

Figure 2: The modelled central Milton Keynes urban area. Avebury, Midsummer, and Silbury Boulevard are the three parallel road links traversing the area SW to NE.

traffic data from a time/day when the road links were most congested: morning rush hour, between 8am and 9am on a non- holiday weekday. Data has been provided by the Milton Keynes Council, and gathered by sensors distributed in the region between December 2015 and December 2016. Traffic signal control information has been provided again by the Council. The model has been calibrated and validated.

During the modelled period, 1,900 vehicles enter the controlled region either to navigate through it, or for reaching one of the parking slots in the area. No vehicle is initially in the area, but they are all injected over the simulation time. All vehicles have a pre-defined destination and route. The largest flow of traffic comes from the west entry points, via the large North Grafton roundabout (the largest roundabout of the map). Many residential areas are connected with the centre of Milton Keynes through those entry points.

The approaches presented in the previous section have been implemented in Python, and use the TRaCI interface to interact with the SUMO simulation environment, in order to get the current network status, communicate with approaching vehicles, and inform vehicles of re-routing. As lookahead time $T$ we considered 10 minutes; this has been observed to be sufficient to allow vehicles entering the network to reach their destination under normal traffic conditions. For every origin-destination, between 2 and 3 alternative routes are considered for distributing traffic. Such routes have been provided by a human expert. While alternatives can, in principle, be automatically calculated (see, for instance, [12]), relying on human expertise can allow to exploit some insights that are based on knowledge that is not captured by the symbolic model of the network. The simulation is run for 1 hour and then stopped. For each set of experiments, the simulation is run five times and results are averaged, to account for non-determinism.

\section{B. Results}

In a first set of experiments, we consider the ideal scenario: all the vehicles that are navigating through the network are connected vehicles, and follow the re-routing instructions provided by either the centralised controller or their own decentralised navigation system. The results of this analysis are presented in Figure 3, in terms of number of vehicles that reached their destination during the considered time window (left), and in terms of average travelling times (right). Presented results include the performance reached by the network when: no traffic optimisation system is in place (default, black); the introduced centralised approach is used (centralised, light blue), and the decentralised optimisation is considered (decentralised, light green). As expected, both the approaches are significantly improving the performance of the network, when compared to the default strategy. Generally, the decentralised technique shows slightly better performance: it allows a few more vehicles to reach their destination in the considered time window, and is generally able to maintain the average travel time a few tens of seconds lower than the centralised approach. Our intuition is that the proposed centralised approach may have a too pessimistic view of the network conditions in some cases. The notion of cumulative occupancy can lead to situations where alternatives are all considered equal by the traffic control system, due to the fact that the network, or a subpart of it, is perceived as very congested -even though only because of vehicles that are predicted to travel through it in the future. This can result in an ineffective re-routing.

To gain some insights into the robustness of centralised and decentralised approaches for traffic optimisation, we run a set of experiments that focused on assessing the importance of penetration rate. We considered cases where $50 \%, 30 \%$, and $10 \%$ of the vehicles are following the instructions provided. Figure 4 reports the impact of the different considered penetration rates on the centralised (left) and decentralised (right) approaches in terms of vehicles that reach their destination in the considered time window. Figure 5 gives the results in terms of average travel time. Interestingly, the decentralised approach requires a very high penetration rate to outperfom the centralised approach. In fact, the decentralised approach outperforms the centralised technique only when $100 \%$ of the vehicles are following the provided instructions. Our intuition is that when all vehicles follow the re-routing instructions, the decentralised approach can balance the use of the network, and vehicles are evenly spread. In ideal conditions, the decentralised approach is basically controlling the evolution of the network conditions -even though in an indirect way. Instead, when only part 

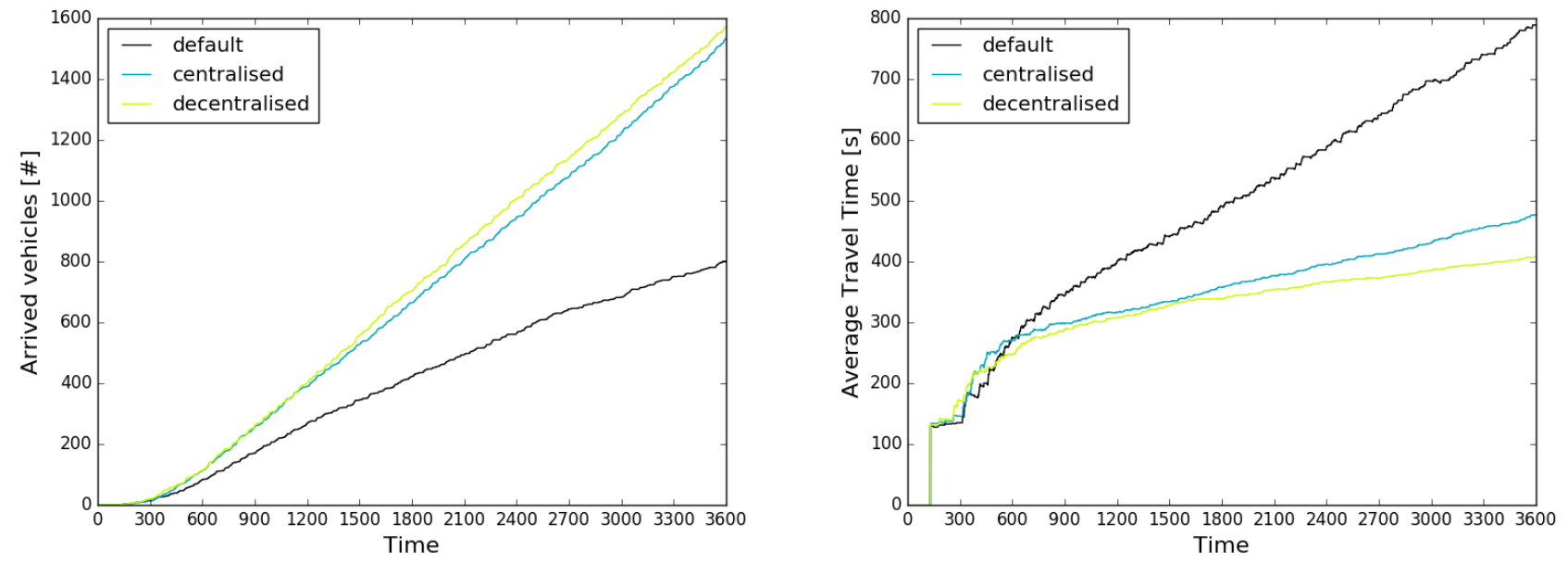

Figure 3: A comparison between the considered centralised and decentralised approaches, in terms of number of vehicles that reached their destination (left) and average travel time (right). Default indicates the performance of the network when no control is in operation, i.e. vehicles are following their pre-calculated routes to reach their destinations.
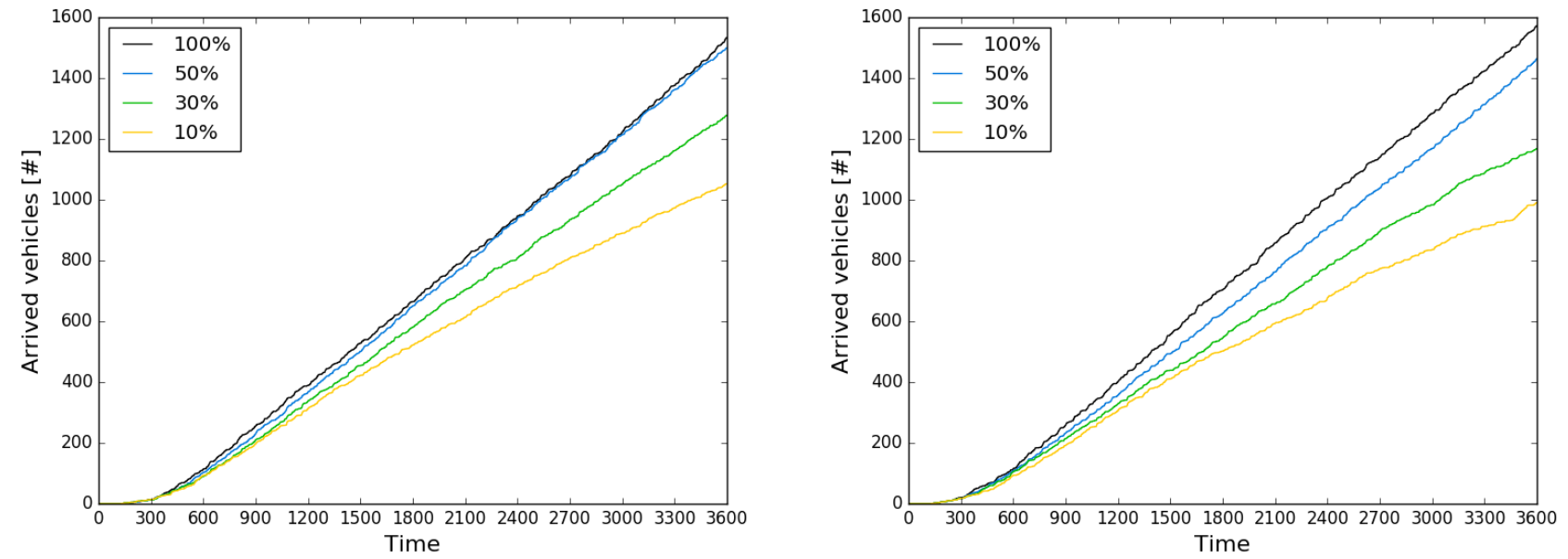

Figure 4: Impact of the penetration rate on the number of arrived vehicles for the centralised approach (left) and the decentralised approach (right).

of the vehicles are following the given instructions, the decentralised approach tends to re-route all the vehicles via the same "alternate" path, leading to congestion.

Notably, the centralised approach is very robust with regards to the penetration rate. In the considered urban region, even a $50 \%$ penetration rate allows the centralised technique to deliver performance that are very close to those achievable with a $100 \%$ rate. This suggests that, while the decentralised approach can better optimise the traffic distribution in ideal conditions, a centralised technique can leverage on its knowledge of the current and predicted network conditions to maximise the impact even if a limited percentage of vehicles is following the re-routing sugges- tions.

Finally, with the aim of evaluating the two approaches in presence of extreme traffic flows, we consider a scenario where the amount of vehicles that enter the network during the modelled time period is doubled. Results, in terms of vehicles that reach their destination, are presented in Figure 6. The first notable aspect to consider is that, if no traffic optimisation approach is in place, the performance of the network drastically deteriorates. By comparing Figure 6 and Figure 3 (left), it is possible to notice that the number of vehicles reaching their destination during the considered time windows drops from approx. 800 to 450 . In other words, the network is extremely congested, and a large number of 

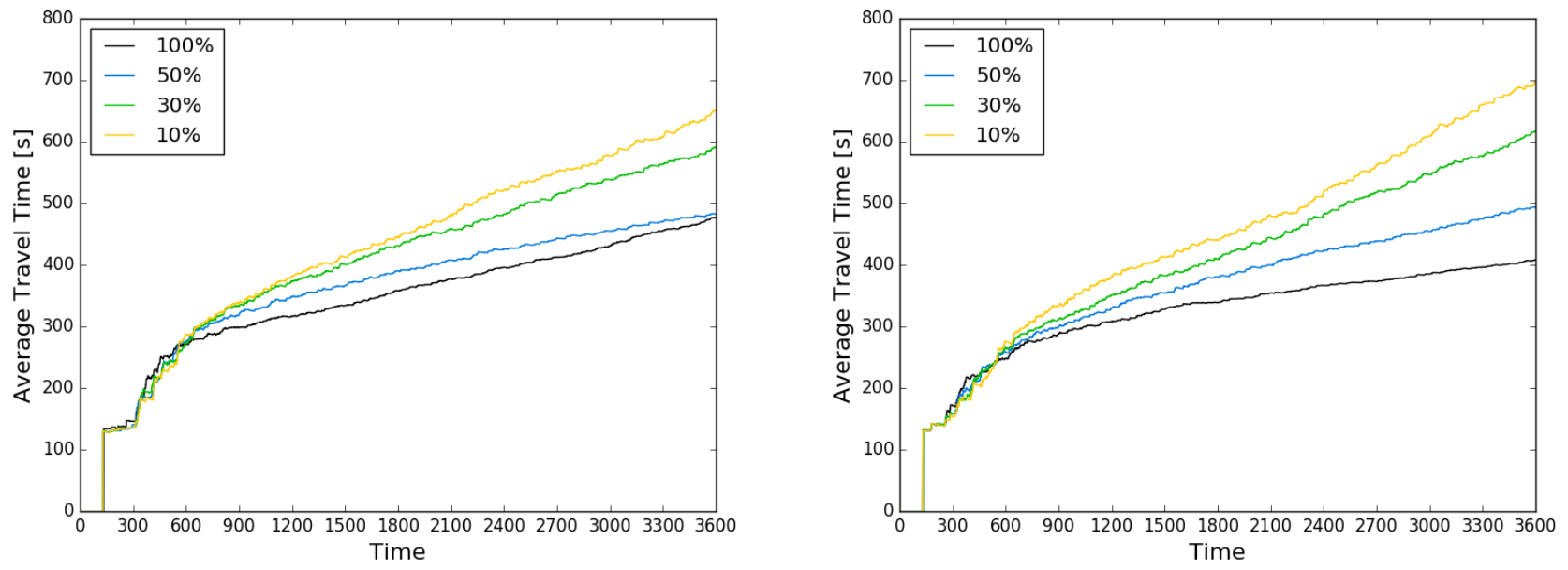

Figure 5: Impact of the penetration rate on the average travel time of vehicles navigating through the controlled region, when using the centralised approach (left) or the decentralised approach (right).

links are blocked. On the contrary, both the centralised and the decentralised techniques for traffic optimisation allow to maintain a good level of service, and a larger number of vehicles is able to reach their destination. In this extreme scenario, the two approaches perform similarly, with the decentralised approach that delivers more vehicles between 1,200 and 2,400 simulation seconds, and the centralised approach that improves later in the scenario.

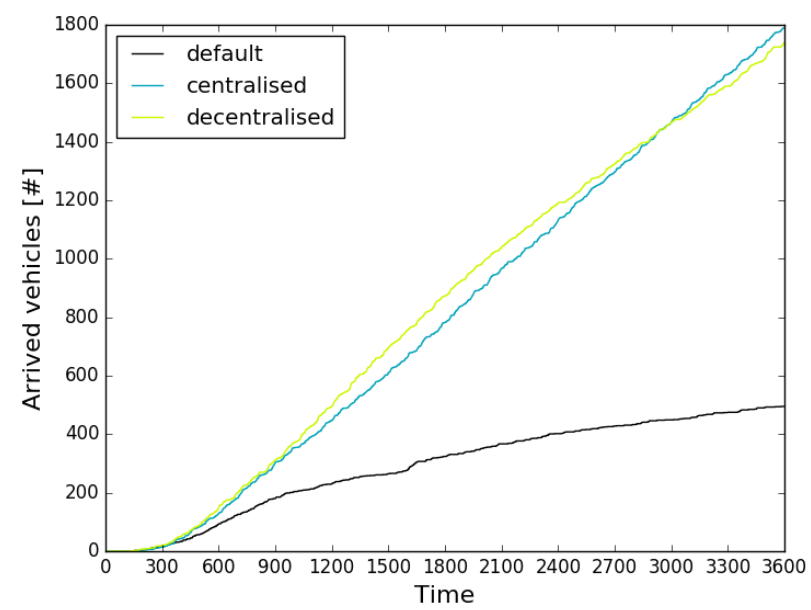

Figure 6: The number of vehicles that reach their destination, during the considered time window, when the amount of traffic is doubled.

\section{CONCLUSIONS}

This paper compares under realistic traffic conditions two approaches for performing traffic optimisation in presence of connected vehicles. One approach, that is decentralised in nature, is based on the traditional technique exploited by available satellite navigators; a vehicle decides in isolation the route to follow given the current travel times of the network links. The other approach, that has been introduced in this paper, takes a centralised perspective and re-routes vehicles by taking advantage of the notion of cumulative occupancy of the links in the controlled region, that is a computationally cheap prediction of future evolution of network conditions. We compared the approaches in simulation using real-world data (and an extreme traffic scenario) of the morning peak hour of a large UK town.

Overall, the simulation results indicate that use of traffic optimisation approaches, either centralised or decentralised, can significantly improve the traffic condition of a urban region during busy periods -such as the morning peak hour. The decentralised strategy demonstrates to perform well when a very high percentage of vehicles are following the provided re-routing instructions. In other words, decentralisation seems to work well when it is exploited by most of the vehicles. The centralised approach is instead more robust, and can improve the traffic conditions also in cases where the penetration rate is limited. Further, both the approaches demonstrated to be able to cope with very high flows of traffic.

Notably, the introduced centralised approach focused on minimising the computational complexity on the urban traffic controller side, and in minimising the exchange of information between vehicles and controller. More sophisticated approaches can consider a richer set of features for characterising the network conditions, and can also take into account traffic light settings, in order to accurately estimate journey times and routes congestion.

With regards to the results of our analysis, we are interested in investigating the performance of centralised and 
decentralised approaches in different urban areas, and we are actively engaging with traffic authorities for performing field trials in some selected regions. That would allow to derive more comprehensive conclusions.

\section{ACKNOWLEDGEMENT}

Research was partially funded by the EPSRC grant EP/R51343X/1 (AI4ME).

\section{REFERENCES}

[1] M. Vallati and L. Chrpa, "A principled analysis of the interrelation between vehicular communication and reasoning capabilities of autonomous vehicles," in 21 st International Conference on Intelligent Transportation Systems, ITSC, 2018, pp. 3761-3766.

[2] F. Arena and G. Pau, "An overview of vehicular communications," Future Internet, vol. 11, no. 2, p. 27, 2019.

[3] D. Manolis, T. Pappa, C. Diakaki, I. Papamichail, and M. Papageorgiou, "Centralised versus decentralised signal control of large-scale urban road networks in real time: a simulation study," IET Intelligent Transport Systems, vol. 12, no. 8, pp. 891-900, 2018.

[4] K. Ramamohanarao, J. Qi, E. Tanin, and S. Motallebi, "From how to where: Traffic optimization in the era of automated vehicles," in Proceedings of the 25th ACM SIGSPATIAL International Conference on Advances in Geographic Information Systems, 2017.

[5] F. Jimoh, L. Chrpa, T. McCluskey, and M. M. S. Shah, "Towards application of automated planning in urban traffic control," in 2013 16th International IEEE Conference on Intelligent Transportation Systems (ITSC 2013). Institute of Electrical and Electronics Engineers ( IEEE ), 2013, pp. 985-990.

[6] L. Chrpa, D. Magazzeni, K. McCabe, T. L. McCluskey, and M. Vallati, "Automated planning for urban traffic control: Strategic vehicle routing to respect air quality limitations," Intelligenza Artificiale, vol. 10, no. 2, pp. 113-128, 2016.

[7] L. Chrpa, M. Vallati, and S. Parkinson, "Exploiting automated planning for efficient centralized vehicle routing and mitigating congestion in urban road networks," in Proceedings of the 34th ACM/SIGAPP Symposium on Applied Computing, SAC 2019, Limassol, Cyprus, April 8-12, 2019, 2019, pp. 191-194.

[8] J. Jeong, H. Jeong, E. Lee, T. Oh, and D. H. Du, "Saint: Self-adaptive interactive navigation tool for cloud-based vehicular traffic optimization," IEEE Transactions on Vehicular Technology, vol. 65, no. 6, pp. 4053-4067, 2015.

[9] Y. Shen, J. Lee, H. Jeong, J. Jeong, E. Lee, and D. H. $\mathrm{Du}$, "Saint+: Self-adaptive interactive navigation tool+ for emergency service delivery optimization," IEEE Transactions on Intelligent Transportation Systems, vol. 19, no. 4, pp. 1038-1053, 2017.

[10] M. Rezaei, H. Noori, D. Rahbari, and M. Nickray, "Refocus: A hybrid fog-cloud based intelligent traffic rerouting system," in 2017 IEEE 4th International Conference on Knowledge-Based Engineering and Innovation (KBEI). IEEE, 2017, pp. 0992-0998.
[11] P. A. Lopez, M. Behrisch, L. Bieker-Walz, J. Erdmann, Y.-P. Flötteröd, R. Hilbrich, L. Lücken, J. Rummel, P. Wagner, and E. Wießner, "Microscopic traffic simulation using sumo," in 21 st International Conference on Intelligent Transportation Systems, ITSC, 2018.

[12] I. Abraham, D. Delling, A. V. Goldberg, and R. F. Werneck, "Alternative routes in road networks," Journal of Experimental Algorithmics (JEA), vol. 18, pp. 1-1, 2013. 